\title{
Measuring the orbital angular momentum of partially coherent optical vortices through singularities in their cross-spectral density functions
}

\author{
Yuanjie Yang, ${ }^{1,2}$ Michael Mazilu, ${ }^{2, *}$ and Kishan Dholakia ${ }^{2}$ \\ ${ }^{1}$ Institute of Astronautics \& Aeronautics, University of Electronic Science and Technology of China, Chengdu 610054, China \\ ${ }^{2}$ SUPA, School of Physics and Astronomy, University of St. Andrews, North Haugh, St. Andrews KY16 9SS, UK \\ *Corresponding author: michael.mazilu@st-andrews.ac.uk
}

Received September 3, 2012; revised October 22, 2012; accepted October 24, 2012; posted October 24, 2012 (Doc. ID 175503); published November 28, 2012

\begin{abstract}
By analysis of the far-field cross-correlation function (CCF) of partially coherent optical beams, we demonstrate an implicit rule that the number of ring dislocations (dark zones) of the far-field CCF is equal to the original topological charge of an optical vortex rendered partially coherent. This novel link between an optical vortex and its correlation singularity may offer an efficient method for measuring the orbital angular momentum of partially coherent optical vortices in fields such as astrophysics and astronomy, as well as atmospheric laser communication. @ 2012 Optical Society of America

OCIS codes: $030.0030,050.4865$
\end{abstract}

It is well known that an optical vortex with a phase distribution of the form $\exp (\mathrm{im} \phi)$ may carry orbital angular momentum (OAM) of $m \hbar$ per photon, where $m$ is an integer number and denotes the topological charge (azimuthal index) of the field [1]]. In the past two decades, OAM has found numerous applications, including optical manipulation [2,3] , quantum information processing, and quantum cryptography [4-6]. More recently, OAM has seen increasing applications in free-space information transfer and communications $[7,8]$. On the other hand, the interest for OAM of light in astronomy and astrophysics has grown $[9,10]$. Optical vortices generated from starlight beams have been observed at the Asiago $122 \mathrm{~cm}$ telescope [9], and the properties of optical vortices have found interesting applications in astronomical coronography [10]. The measurement of the azimuthal index $m$ associated with a given vortex field is crucial for all these fields and may yield interesting new studies in astrophysics [11].

Determining the azimuthal index $m$ of a vortex remains an intriguing problem in both the quantum and the classical domains [12]. For classical light fields, an array of various diffractive apertures have been used in recent years: in 2008, Berkhout and Beijersbergen [11] probed the OAM of optical vortices by analyzing the far-field diffraction intensity patterns after a vortex propagates through a multipoint interferometer. Other methods have also been introduced to measure the OAM of optical vortices based on the diffraction phenomena [12-14]. More recently, it has been shown that a triangular aperture illuminated with a vortex beam creates a truncated lattice diffraction pattern and that the resultant diffraction pattern can be used to measure the OAM of light beams [15-17]. Other methods for measuring the topological charge of an optical vortex have included the diffraction intensity pattern after an axicon [18] or an annular triangle aperture [19]. Very recent studies have shown the use of multivariate statistical analysis for the diffraction pattern from any arbitrary aperture and shown that by training the system one may deduce the index $m$ of the vortex from the diffraction pattern of any aperture [20]. While some of above methods may deal with vortices generated with temporally incoherent light, it is noteworthy that all of these methods have assumed that the optical field is fully spatially coherent. However, typically the field may be partially spatially coherent under usual circumstances, and the aforementioned methods are not necessarily readily amenable for the case of a partially coherent incident vortex field.

The last few years have seen some interesting studies based upon the spatial correlation functions of a partially coherent light field. These may possess phase correlation singularities denoting nulls of the cross-spectral density function. The singularity in such a function thus shows regions where the phase is ill-defined. While some studies have been performed showing the use of such correlation vortices [21-23], no study has yet made a definitive linkage between the azimuthal index of an optical vortex and the vortices present in the cross-correlation function (CCF) and exploited this relationship.

In this Letter, we explore the azimuthal mode index of partially coherent optical vortices. In particular, we determine the CCF of such light fields. Investigating the relationship between an optical vortex and its CCF reveals a method by which we may infer the azimuthal index for a spatially incoherent incident light field where the spatial coherence dimension of the field is comparable to the beam-waist size.

Consider a partially coherent beam whose mutual coherence function (MCF) at the source plane $z=0$ can be written as [21]

$$
\begin{aligned}
\Gamma\left(\vec{\rho}_{1}, \vec{\rho}_{2}, 0\right)= & E_{0}\left(\vec{\rho}_{1} \cdot \vec{\rho}_{2}\right)^{m} \exp \left(-\frac{\left|\vec{\rho}_{1}-\vec{\rho}_{2}\right|^{2}}{L_{c}^{2}}\right) \\
& \times \exp \left(-\frac{\rho_{1}^{2}+\rho_{2}^{2}}{w_{0}^{2}}\right) \exp \left(-i m\left(\phi_{2}-\phi_{1}\right)\right),
\end{aligned}
$$

where $E_{0}$ is a constant, $m$ is topological charge of the vortex, $L_{c}$ is the transverse coherence length, $w_{0}$ denotes the waist width, and $\vec{\rho}_{1}, \vec{\rho}_{2}$ and $\phi_{1}, \phi_{2}$ are the transversal position vector and azimuthal coordinates in the cylindrical coordinate system. 
In the far field, the MCF is given by [21]

$$
\begin{aligned}
\Gamma\left(\vec{\rho}_{1}, \vec{\rho}_{2}, z\right)= & \left(\frac{1}{\lambda z}\right)^{2} \iiint \int \mathrm{d} \vec{\rho}_{1}^{\prime} \mathrm{d} \vec{\rho}_{2}^{\prime} \Gamma\left(\vec{\rho}_{1}^{\prime}, \vec{\rho}_{2}^{\prime}, 0\right) \\
& \times \exp \left(-\frac{2 \pi i}{\lambda z}\left(\vec{\rho}_{1}^{\prime} \cdot \vec{\rho}_{1}-\vec{\rho}_{2}^{\prime} \cdot \vec{\rho}_{2}\right)\right),
\end{aligned}
$$

where $\lambda$ is the wavelength.

From Eq. (2), we may determine the far-field CCF $\chi_{c}(\vec{\rho})=\Gamma(\vec{\rho},-\overrightarrow{\vec{\rho}})$ and the intensity (the autocorrelation function) $\chi_{a}(\vec{\rho})=\Gamma(\vec{\rho}, \vec{\rho})$, and this is shown in Fig. 1 , for the case of topological charge $m=3$. From Fig. 1(a) we can see that for the high coherence case $\left(L_{c}=3 w_{0}\right)$ there is a dark vortex core with a minimum intensity. Figures 1(a)-1(c) show that the dark vortex core of intensity fills with diffuse light as the coherence decreases, a feature well established in the literature. However, interestingly bright and dark rings appear in the CCF, and the central region of the CCF increases in intensity as the spatial coherence is decreased, as shown in Figs. 1(d) $-1(\mathrm{f})$. Moreover, it is noted that the number of the dark rings present is three, the very value of the original topological charge of the field.

To see the effect of spatial coherence on the far-field CCF more clearly, we can assume $m=1$, and the far-field CCF can be written in closed form:

$$
\begin{aligned}
\chi_{c}(x, y)= & \left(2 z^{2} \beta-k^{2}\left(x^{2}+y^{2}\right)\left(k^{2} w_{0}^{4} T_{c}^{4}+4 z^{2} \alpha^{2}\right)\right) \\
& \times \frac{k^{2} w_{0}^{8} T_{c}^{2}}{\beta^{3}} \exp \left(-\frac{2 k^{2} \alpha w_{0}^{2}\left(x^{2}+y^{2}\right)}{\beta}\right),
\end{aligned}
$$

where

$$
\alpha=w_{0}^{2}+T_{c}^{2},
$$
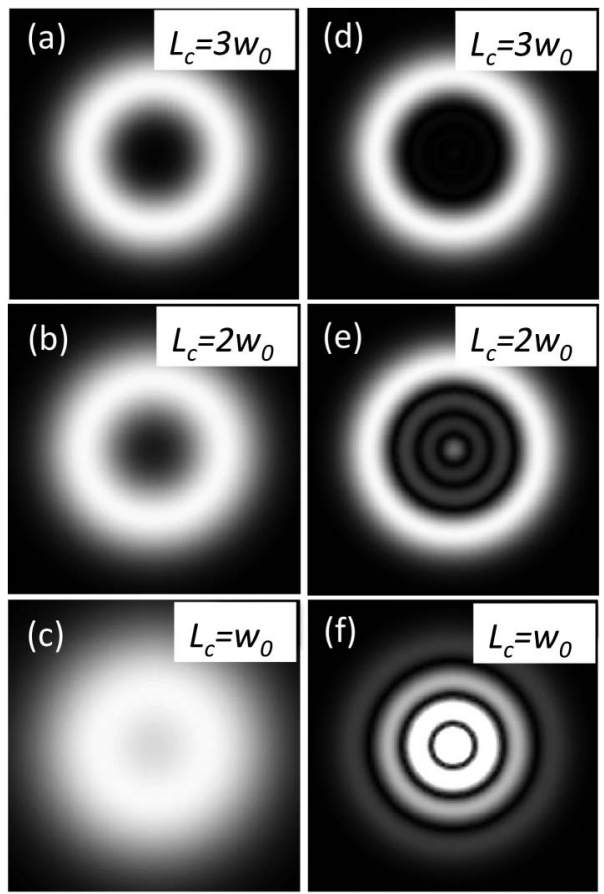

Fig. 1. Far-field intensity (a)-(c) and cross-correlation functions (CCFs) (d)-(f) for $m=3$ for coherence lengths $L_{c}$.

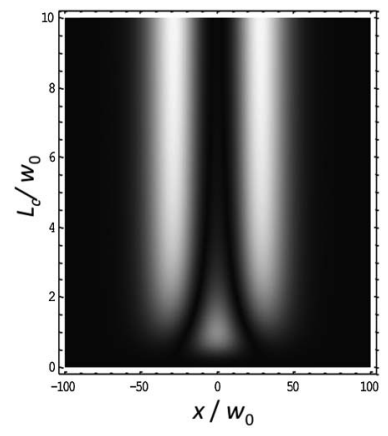

Fig. 2. One-dimensional far-field CCFs influenced by coherence lengths $L_{c}$, where $m=1$.

$$
\beta=4 z^{2} \alpha+k^{2} w_{0}^{4} T_{c}^{2}
$$

From Eq. (3) we can determine the one-dimensional CCF and the influence of the spatial coherence, and this is shown in Fig. 2. From Fig. 2 we can see that for the low coherence case, the center CCF is maximum, and there is a dark zone. Moreover, the radius of the dark zone decreases gradually with the increasing coherence, and the CCF has an in-axis null when the coherence is bigger a certain value, as shown in Fig. 1(a).

To determine the analytical form of the far-field CCF for higher-order vortices, we decompose the integral into a part that depends on $x_{1}$ and $x_{2}$ multiplied by one that depends on $y_{1}$ and $y_{2}$. For each of these parts, we expand the vortex parts into a higher-order polynomial and treat each individual component using the following definition:

$$
\begin{aligned}
P_{i j}= & \iint \mathrm{d} x_{1} \mathrm{~d} x_{2} x_{1}^{i} x_{2}^{j} \\
& \times \exp \left(b_{1} x_{1}^{2}+c_{1} x_{1}+b_{2} x_{2}^{2}+c_{2} x_{2}+a x_{1} x_{2}\right) .
\end{aligned}
$$

The zeroth-order integral can be readily determined as

$$
P_{00}=\frac{2 \pi}{\sqrt{4 b_{1} b_{2}-a^{2}}} \exp \left(-\frac{b_{1} c_{2}^{2}+b_{2} c_{1}^{2}-c_{1} c_{2} a}{4 b_{1} b_{2}-a^{2}}\right),
$$

where the coefficients $\left(a, b_{1}, c_{1}, b_{2}, c_{2}\right)$ are chosen such that the integral converges. Higher-order terms can be deduced by using the following recurrence relationships:

$$
P_{i j}=\frac{\partial^{i} \partial^{j} P_{00}}{\left(\partial c_{1}\right)^{i}\left(\partial c_{2}\right)^{j}}
$$

where each partial derivative with respect to $c_{1}$ and $c_{2}$ corresponds to the multiplication with $x_{1}$ and $x_{2}$, respectively, in Eq. (6).

The far-field CCF with different topological charges for the partial and low-coherence cases is shown in Fig. 3. From these results and from further simulations not shown here, we can see the same phenomena, i.e., the number of dark-ring dislocations in the far-field CCF is equal to the topological charge of the partially coherent optical vortex. Therefore, one can measure the topological charge of partially coherent optical vortex through its 

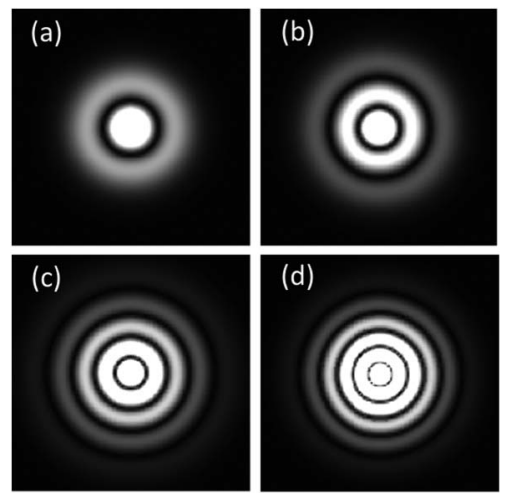

Fig. 3. Cross-correlation functions (CCFs) in the far-field plane for different topological charges, where $L_{c}=w_{0}$. (a) $m=1$, (b) $m=2$, (c) $m=4$, (d) $m=5$.

far-field CCF using, for example, an experimental system similar to that demonstrated elsewhere [21].

To understand how the topological charge (azimuthal index) of the vortex beam becomes mapped onto the number of rings in the far-field CCF, we can visualise the different transformations involved in its definition. Indeed, the integral corresponds to the cross-correlation between the fields diametrically opposed with respect to the vortex line position. This cross correlation involves only two points in the case of perfectly coherent beams. Partially coherent beams deliver a cross correlation over larger regions, depicted by the yellow circles in the associated video. In fact, we find that the size of the region is inversely proportional to the coherence length. It is actually this coherence length that determines the length scale over which the CCF integrates the product between the fields on either sides of the vortex. As the distance between the two diametrically opposed regions is increased, we observe a number of constructive and destructive interference features in the CCF. These are due to the variation of the number of $2 \pi$ phase changes within the yellow region. As the size of the region is constant, this number varies from $2 m \pi$ to 0 as the region moves from the center of the beam to the outside of the beam. This variation delivers altogether $m$ ring dislocations and one central spot. We remark that our approach is not restricted to the case of Laguerre-Gaussian modes but should have wider applicability for the case of nested vortices in other light fields. This is the topic of further study.

In conclusion, the general relationship between the topological charge and the CCF of partially coherent optical vortices is numerically studied. The numerical simulation reveals that the number of ring dislocations of the far-field CCF of partially coherent optical vortices is just equal to the value of topological charge. Therefore, this shows we may measure the OAM content of a partially spatially coherent vortex field by observing its CCF. This phenomenon is generic and may occur not only in the optical domain but also in any partially coherent vortex wave.

YY acknowledges support by the National Natural Science Foundation of China under grant nos. 61205122 and 61107037 and the Fundamental Research Funds for the Central Universities under grant no. ZYGX2010J112. K. D. is a Royal Society Wolfson-Merit Award holder and thanks the UK EPSRC for funding.

\section{References}

1. L. Allen, M. Beijersbergen, R. Spreeuw, and J. Woerdman, Phys. Rev. A 45, 8185 (1992).

2. H. He, M. Friese, and N. R. Heckenberg, Phys. Rev. Lett. 75, 826 (1995).

3. K. Dholakia and T. Čižmár, Nat. Photonics 5, 335 (2011).

4. A. Vaziri, J. W. Pan, T. Jennewein, G. Weihs, and A. Zeilinger, Phys. Rev. Lett. 91, 227902 (2003).

5. G. Molina-Terriza, A. Vaziri, J. Reháček, Z. Hradil, and A. Zeilinger, Phys. Rev. Lett. 92, 167903 (2004).

6. G. Molina-Terriza, J. P. Torres, and L. Torner, Nat. Phys. 3, 305 (2007).

7. I. B. Djordjevic, Opt. Express 19, 14277 (2011).

8. J. Wang, J.-Y. Yang, I. M. Fazal, N. Ahmed, Y. Yan, H. Huang, Y. Ren, Y. Yue, S. Dolinar, M. Tur, and A. E. Willner, Nat. Photonics 6, 488 (2012).

9. G. Anzolin, F. Tamburini, A. Bianchini, G. Umbriaco, and C. Barbieri, A\&A 488, 1159 (2008).

10. C. Barbieri, F. Tamburini, G. Anzolin, A. Bianchini, E. Mari, A. Sponselli, G. Umbriaco, M. Prasciolu, F. Romanato, and P. Villoresi, Earth Moon Planet 105, 283 (2009).

11. G. C. G. Berkhout and M. W. Beijersbergen, Phys. Rev. Lett. 101, 100801 (2008).

12. G. C. G. Berkhout, M. P. J. Lavery, J. Courtial, M. Beijersbergen, and M. J. Padgett, Phys. Rev. Lett. 105, 153601 (2010).

13. C.-S. Guo, L.-L. Lu, and H.-T. Wang, Opt. Lett. 34, 3686 (2009).

14. C.-S. Guo, S.-J. Yue, and G.-X. Wei, Appl. Phys. Lett. 94, 231104 (2009).

15. J. M. Hickmann, E. J. S. Fonseca, W. C. Soares, and S. Chavez-Cerda, Phys. Rev. Lett. 105, 053904 (2010).

16. L. E. E. de Araujo and M. E. Anderson, Opt. Lett. 36, 787 (2011).

17. A. Mourka, J. Baumgartl, C. Shanor, K. Dholakia, and E. M. Wright, Opt. Express 19, 5760 (2011).

18. Y. Han and G. Zhao, Opt. Lett. 36, 2017 (2011).

19. Y. Liu, J. Pu, and B. Lü, Appl. Opt. 50, 4844 (2011).

20. M. Mazilu, A. Mourka, T. Vettenburg, E. M. Wright, and K. Dholakia, Appl. Phys. Lett. 100, 231115 (2012).

21. D. Palacios, I. Maleev, A. Marathay, and G. A. Swartzlander, Jr., Phys. Rev. Lett. 92, 143905 (2004).

22. Y. Gu and G. Gbur, Opt. Commun. 282, 709 (2009).

23. Y. Gu and G. Gbur, Opt. Commun. 283, 1209 (2010). 